\title{
Designing a Touch-Screen SenseCam Browser to Support an Aging Population
}

Niamh Caprani, Aiden R. Doherty, Hyowon Lee, Alan F. Smeaton, Noel E. O'Connor and Cathal Gurrin

CLARITY: Centre for Sensor Web Technologies Dublin City University

Ireland

ncaprani@computing.dcu.ie

\begin{abstract}
In this paper, we describe the HCI challenges

associated with the novel domain of lifelogging for older users. The SenseCam is a passively capturing wearable camera, worn via a lanyard around the neck and used to create a personal lifelog or visual recording of the wearer's life, which generates information that may be very helpful as a human memory aid. Indeed, given that memory defects are more marked in the elderly, we believe that lifelogging browsing techniques which are considerate of the elderly are imperative. Thus, the challenge tackled in this work was to design and integrate the lifelogging activity supported by new technologies in such a way that can easily be learned and used by older people, enabling them to enhance and enrich their lives with the new technologies. This work provides design practitioners of future lifelogging interfaces early sight of the lessons we have learned in making lifelogging technologies accessible to elderly non-computing literate participants.
\end{abstract}




\section{Keywords}

SenseCam, touch-screen, older adults

\section{ACM Classification Keywords}

H5.2 Information Interfaces and Presentation: User Interfaces - D.2.2, H.1.2, I.3.6

\section{General Terms}

\section{Human Factors}

\section{Introduction}

The SenseCam, developed by Microsoft Research in Cambridge, is a small wearable camera which passively and automatically captures thousands of images per day. For such a large amount of visual information to be easily and efficiently accessed by a user, we utilise an automated technique to structure the photos into 'events', as well as select representative photos to be presented to the user [2]. An event could be eating breakfast, dong the crossword or walking outside. The benefits of SenseCam as a memory retrieval aid are emerging at a rapid pace and the interest in SenseCam is steadily increasing $[1,5]$. Preliminary studies show that the SenseCam has the ability to support episodic memory in individuals, and it has been shown to increase the likelihood that these memories will be retained over time [1].

Although older adults are increasingly becoming more computer literate, it is important for designers to consider how the effects of ageing may influence the way in which older adults interact with systems and devices. Some examples of the changes we experience which have implications on the design of technology are: sensory processes (difficulty adjusting visual focus, problems with visual acuity, a gradual reduction in contrast and colour sensitivity; difficulty hearing higher frequencies); psychomotor processes (depletion in muscle strength and flexibility; physical problems due to stroke, arthritis, or falls) and cognitive processes (gradual depletion of cognitive processing resources; working memory, selective attention, divided attention and the rate of processing with increased task complexity all show age-related declines; older adults are slower to acquire and maintain new procedures than younger adults) $[3,4,7]$.

\section{The development of the Sensecam browser}

Purpose of Current Study

The purpose of this study was to design a browser using novel technologies to facilitate older people, who had never used a computer, to easily review their

SenseCam images. The proposed SenseCam browser was developed through three stages:

1. An evaluation of two existing SenseCam browsers

2. The design of a new SenseCam browser

3. The evaluation of the new SenseCam browser through a longitudinal field study with older users

\section{Evaluating Existing SenseCam Browsers}

As part of our work we initially asked three older adults between the ages of 62 and 79 years to assess two SenseCam image browsers currently being used by neuropsychological researchers, (1) DCU event segmentation browser [6] and (2) Microsoft SenseCam browser [5].

The participants wore the SenseCam over a 2 day period and then reviewed the images using both aforementioned browsing applications, one after the other in alternating sequence. Questions were put to 
the participants regarding the physical characteristics of the browsers (screen characters, icons and text),

navigation (how to view next/previous images, different days), organisation of information (natural grouping of information, consistency), and conceptual (feedback to user, system status, possibility for error making). Some of the common problems that were identified from these browsers were as follows:

- Image thumbnail size too small to identify context

- Speed of image presentation too fast to process

- Text size and colour contrast not appropriate

- Scrolling caused difficulties

- Computer terms were not easily understood

- Event information was not clear (time and date)

- No help option available

- Difficulty navigating to different days or time of day

- Require experience using desktop computer

\section{Suggested design}

The issues that emerged from this exploratory study were used to guide the design of a new SenseCam image browser for older adults. Throughout the design process, low fidelity prototypes were shown to older individuals to obtain continuous feedback and to keep the design focus on older users. The final prototype design can be seen in Figure 1 . The factors implemented into the design were as follows:

Physical characteristics

- The application was designed for touch-screen use to encourage novice computer usage
- On the main screen, event keyframe images ${ }^{1}$ are displayed large enough to recognise context

- Target buttons are designed to be large to encourage usability and interaction

- Text size is set at a minimum of 14 pt to accommodate visionary difficulties

- There is a focus on high colour contrast between the text and background colour

- The image size was set so that the content and context can be easily recognised by users

Navigation

- There is no scrolling required throughout the application, instead previous/next buttons are used

- Items are positioned so that they can be easily seen and accessed

- Instructions are provided on each page to guide the user through any action

- Images can be viewed as a continuous slideshow at moderate speed or by looking through them one by one

Information organisation

- The design and layout of each page is standard

- Users have the option to add a label to their event

- Users can readily see information about the images

- Help option highlighted in red to ensure visibility

Conceptual

- Immediate feedback is offered through highlighting and a low frequency beep tone on target selection

- Users are provided with delete and undo delete functionality to improve user control over the content

- The language used is generalized to be understood by novice computer users

${ }^{1}$ An image that represents the user's event. 


\section{User trial and evaluation}

To evaluate whether the design of the SenseCam browser is appropriate for older adults we placed a touch-screen computer with the installed application in the homes of three older participants (different to the original three participants) for a two week period. The participants were between the ages of 64 and 79 years. None of the participants had ever used a computer. The participants were asked to wear the SenseCam everyday and to upload their images using the

SenseCam browser. As the participants had never used a computer, written instructions were provided to guide them through turning on and off computer. The participants were also given an introduction and demonstration of the browser and directed to the help section should they need it. The participants were visited 3-4 times during the trial period.

\section{Data gathering methods}

1. A pre-trial questionnaire was supplied to gather some demographic information

2. The participants were asked to keep a diary

3. The participants' use of the browser was recorded including time interacting with it, buttons pressed etc.

4. A post-trial questionnaire was administered

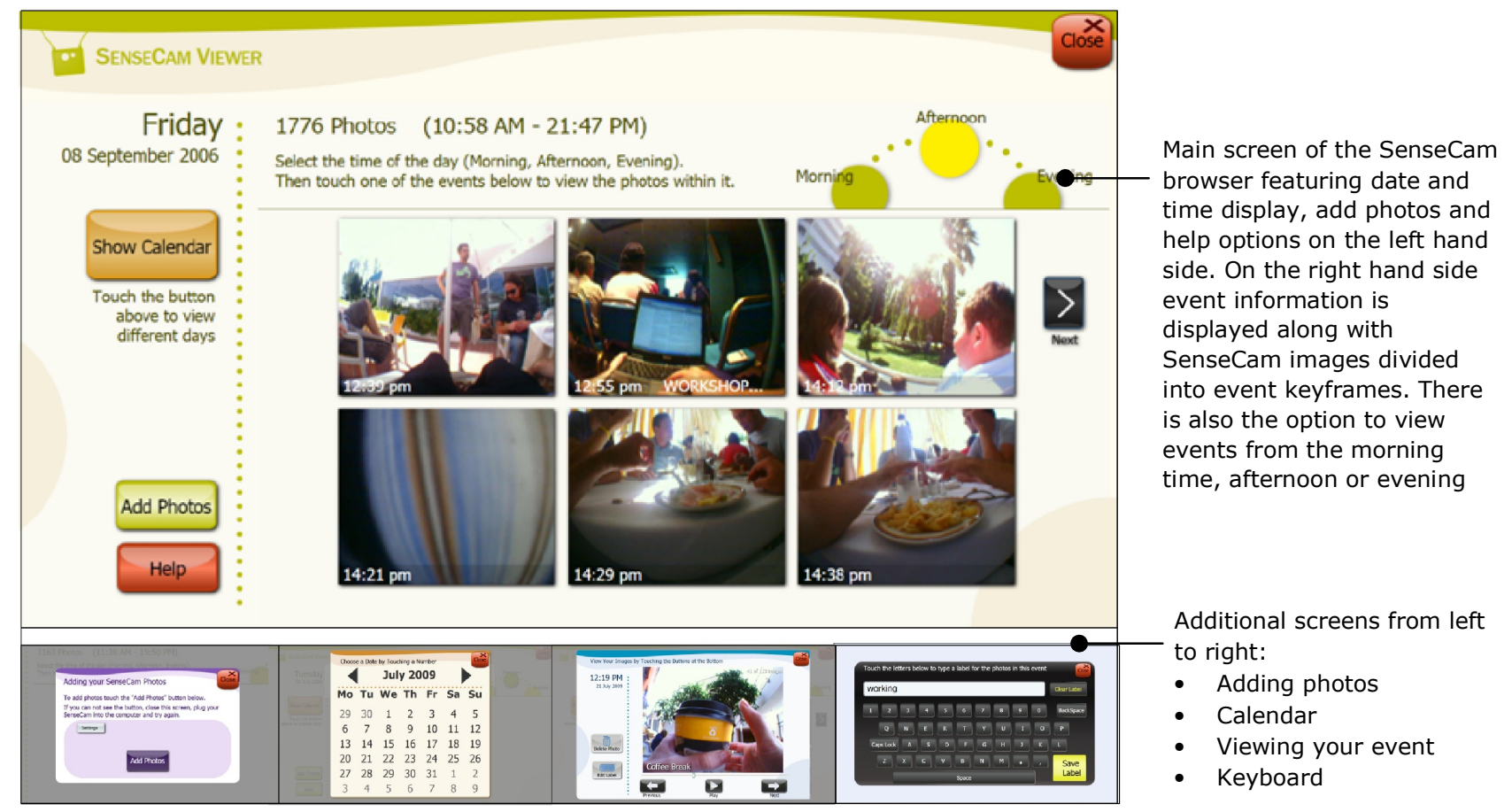

figure 1. The main screen of our proposed SenseCam browser with thumbnails of additional screens below 


\section{Findings}

The findings from the evaluation were taken from the post-trial questionnaire and the automatically recorded interactions. Although the participants were encouraged to write down any problems or thoughts into the diary provided, none of the participants used it. During the trial period some technical issues arose for two of the participants. Only one week of one of the participant's recorded interactions could be used.

The findings show that the participants accessed the SenseCam browser at least once a day throughout the trial period and spent between 15 minutes to 1 hour interacting with it. There were no set times of the day that the participants used the browser. Access to the system was dependant on when the user was in the home and had some free time. In all, our participants collected five week's worth of SenseCam data, corresponding to 27,212 images which were automatically segmented into 308 events. SenseCam data was gathered at regular intervals and our participants interacted with the browser just as frequently.

Novel activity of reviewing the day

Considering the fact that reviewing one's day with passively-captured photos is quite a novel activity that none of our participants has done (or was aware of) before, a number of novel experiences were reported, discussed, and their value was mentioned. For example, one user remarked on how he had taken some good photos of his grandchild at the beach. This example highlights how valuable the SenseCam is for capturing typical day-to-day activities that would not normally be recorded.
Developing personal pattern of use

From the usage data we collected it was revealed that certain features such as the delete and label function, while rarely used by our participants, were perceived as highly useful. Other features such as the buttons to view the different times of day, and the play/pause button were frequently used by our participants as they produce an immediate and obvious output to the user. This highlights how certain features could be perceived more or less important depending on the duration of expected usage, and a longitudinal study such as this should take this into account.

\section{Ease of use}

The users commented that they found the photos to be easy to view and find, and that the browser helped

them to remember what they did on different days. All three of the participants said that they would use the browser again and that they would recommend it to a friend. In particular, the users rated the browser highly for its ease of use, efficiency in accessing information and as an aesthetically pleasing interface. Our

participants found no problems reading the text on the screen and frequently referred to the date of the images being reviewed. The participants also noted that the image size of the thumbnails on the main screen was a good size for them.

Increasing User Confidence in Technology

The participants' confidence in using the touch-screen computer and interacting with the browser gradually increased throughout the trial period. They also found it satisfying to use and felt it gave a sense of future technology. At the beginning of the trial the participants did not know how to turn on or off the computer or how to select an item on the screen. A 
combination of face to face guidance, paper instructions of how to access the computer and browser and the help section within the browser assisted in building up the users' comfort and confidence with the technology. After the trial, all of the participants were accessing and interacting with the system without any input from the researcher: "It took me a while to get used to it as first as I had never used a computer but I soon got in on it".

\section{Conclusions}

We have experienced and discovered numerous issues in introducing lifelogging technologies to elderly nontechnical participants, whom we believe are

representative of a set of users who may find memory aids such as the SenseCam to be of great benefit to their quality of life. There are a number of challenges moving into the future. As images are captured on an external device (to the PC), it is a little daunting for non-technical users to download images from the camera, and we believe that this process must be simplified even further in future. However, we received very positive feedback from our non-technical older participants, who repeatedly commented that they felt they were satisfied with using our browser, and that it gave them a sense of future technology. It was very pleasing to see these participants independently browse their own SenseCam images, and mostly to see their increased sense of confidence in computing technology.

\section{Acknowledgments}

The authors wish to acknowledge the support of

Microsoft Research (grant number 2007-056), and Science Foundation Ireland (grant number 07/CE/I1147).

\section{References}

[1] Berry, E., Kapur, N, Williams, L., Hodges, S., Watson, P., Smyth, G., Srinivasan, J. et al. (2007). The use of a wearable camera, SenseCam, as a pictorial diary to improve autobiographical memory in a patient with limbic encephalitis: A preliminary report. Neuropsych Rehab, 17(4/5), 582-601.

[2] Doherty A.R. \& Smeaton A.F. (2008). Automatically segmenting lifelog data into events. In WIAMIS

Workshop on Image Analysis for Multimedia Interactive Services, Klagenfurt, Austria, 2008.

[3] Fisk, A., Rogers, W. A. Charness, N., Czaja, S. J., \& Sharit, J. (2009). Designing for older adults: Principles and creative human factors approaches $\left(2^{\text {nd }} \mathrm{ed}\right)$. New York: CRC Press.

[4] Hawthorn D. (2000). Possible implications of aging for interface designers, Interacting with Computers, 12, 507--528.

[5] Hodges, S., Williams, L., Berry, E., Izadi, S., Srinivasan, J., Butler, A., Smyth, G et. al. (2006). SenseCam: A retrospective memory aid. In UbiComp: 8th International Conference on Ubiquitous Computing, volume 4602 of LNCS, pp 177-193. California, USA: Springer.

[6] Kumpulainen, S., Jarvelin, K., Serola, S., Doherty A.R., Byrne, D., Smeaton, A.F., \& Jones, G.J.F. (2009) Data collection methods for analyzing task-based information access in molecular medicine. In

MobiHealthInf 2009 - 1st International Workshop on Mobilizing Health Information to Support Healthcarerelated Knowledge Work, Porto, Portugal, 16 January 2009.

[7] Park, S., O'Brien, M.A., Caine, K.E., Rogers, W.A. Fisk, A.D., Van Ittersum, K., Capar, M., \& Parsons, L.J. (2006). Acceptance of computer technology:

Understanding the user and the organisational

characteristics, Proc. of the Human Factors and Ergonomics Society 50 $0^{\text {th }}$ Annual Meeting, 1478-1482. 